Another important consideration is that such hospitals must be staffed not only with ophthalmic surgeons but also with physicians well-versed in the management of women who have recently been delivered. The Liyerpool experience goes to show that little danger attends the careful transference of the mothers from their homes or from lying-in hospitals. In Liverpool, this is done by arrangement with the city authorities. The point will doubtless be carefully considered in any London scheme.

\title{
The Standardization of the Illumination of Test-cards and Perimeters
}

Volume XV (1917) of the Transactions of the American Ophthalmological Society (noticed elsewhere in this issue) contains the preliminary report of the Committee on Standardization of the Illumination of Test-cards and Perimeters. It is the work of a conference of the joint Committees of the Illuminating Engineering Society and of the American Ophthalmological Society.

As to test-types, it deals with factors which influence visual acuity, as $(a)$ insufficient light upon the test-object; $(b)$ too much light or light wrongly distributed; (c) colour of the light; and (d). the effect of a pupillary diameter different from that under which the glasses prescribed are to be worn. It inquires whether the conditions reasonably attainable in the consulting-room afford the maximum possible visual acuity ; what conditions as to brightnessdistribution in the visual field give the best results; and, finally, as to the relative importance of reproducing the "working " size of the pupil, and how it may be attained if essential. There does not appear to be any critical point at which visual acuity ceases to rise with increase of illumination and begins to decline with further increase. Furthermore, it appears that the brightness of an object may undergo material change without important change in the visual acuity. The standard wax candle and the shadow photometer would seem to be all the photometric equipment necessary for adequately accurate measurement of the illumination of the testcard, the only question remaining being the more or less arbitrary one of selecting the illumination which, on the whole, best fulfills all requirements. The effect of the brightness of the surroundings on visual acuity is summed up by the Committee as follows :-(1) Great excess of brightness in the surroundings over that of the chart will reduce visual acuity. (2) Perfectly dark surroundings may not give as high a value as surroundings of the order of brightness of the test-object. Even when somewhat brighter than the test-object, the bright surroundings have been shown to give an advantage of 5 per cent. over the dark in some cases. A high difference in either 
direction is trying to the observer, and should for that reason be avoided. Translucent test-charts are not recommended for use in a dark room. The choice should be in favour of surroundings of a subdued hue, if coloured at all. It is added that "the chart and the patient are to be so arranged that no light-source, window, or other spot of high brightness, such as wall or ceiling, used as secondary source in indirect and semi-indirect lighting, shall be visible to the patient while he is engaged in observing the test-chart."

As to perimetry, the peculiarities of the periphery of the retina led to consideration of the following factors:-(1) the rapid adaptation of the peripheral retina, owing to which a sensation rapidly loses its characteristic quality while the stimulus continues to act upon it ; (2) the fact that the peripheral retina is peculiarly subject to contrast, so that the same test surface gives different results according to the background upon which it is seen; and (3) the fact must not be overlooked that the results obtained are totally difterent with coloured test-spots of different sizes. Even the central retina fails to recognize colour when the coloured spot is small enough. The perimeters do not agree in so fundamental a dimension as the radius of the arc. Various makers put out test-colours of different hues and shades, which pass current under the same colour designation. The technique by which the colour is presented to the patient is extremely variable. The attention paid to the illumination of the test-spot seems wholly inadequate. When all is said and done, the fact remains : the patient is the one thing that cannot be standardized. It is difficult to ascertain whether the errors of perimetry are chiefly personal or chiefly technical, and the Committee recommends in pursuance of this idea that (1) "it be determined what the effect is of such difterences as obtain under the usual daylight illumination of the perimeter in the illumination of the test-spot in different parts of the visual field"; and (2) "samples of the colours be obtained from the various makers with a view to spectrophotometric or colorimetric examination and intercomparison, and possible adoption of certain ones as standard."

The preliminary report is signed by Drs. W. Zentmayer, G. S. Crampton, and H. M. Langdon.

In the final report we hope the Committee may succeed in fixing a definite standard.

\section{American Board for Ophthalmic Examinations}

We gather from the Annual Report of the American Board for Ophthalmic Examinations that examinations were held in December, 1916, at Memphis, and in June, 1917, in New York City, and that the total number of accepted applications was 117 . 